\title{
Design of a Sliding Mode Controller for a Biochemical Process By Using an Intermediate Output Variable
}

\author{
Mehmet Önder Efe ${ }^{1}$
}

\begin{abstract}
Sliding mode control of a chemical process is considered in this paper. The process is nonlinear and the dynamics is highly involved due to the strong interdependencies between the variables involved. The considered process has two state variables named dimensionless concentration and temperature. The goal is to maintain a desired concentration level by suitably altering the coolant temperature. The design is based on the control of an intermediate variable, which is defined as the fictitious output of the process. If the fictitious output tracks an appropriately scaled command signal, it is guaranteed to observe precise tracking in the time evolution of the concentration. The design is based on the nominal model and tests have been carried out on a highly demanding set of operating conditions. The results have shown that the proposed mechanism successfully drives the dimensionless concentration to its desired level and maintains the temperature bounded. Furthermore, certain degrees of robustness displayed against disturbances and uncertainties is another important merit of the presented control scheme.
\end{abstract}

\section{INTRODUCTION}

Chemical processes are often modeled by few differential and/or difference equations exhibiting a very rich set of dynamical responses. The nonlinear nature of such processes renders the design of the control systems quite involved and once coupled with high performance expectations, exploring the nonlinear nature of the system becomes inevitable. Although it is possible to obtain satisfactory results with linear controllers, addressing the nonlinearity in an appropriate manner enhances the long term profit at large scales and this is generally a challenge due to the uncertainties and impreciseness in the operating environment. From a control engineer's point of view, the control system must be robust against the disturbing factors.

Variable Structure Systems (VSS) with a sliding mode have extensively been studied by many researchers. The invariance properties and insensitivity to parameter variations are the most prominent features that are observed in a variable structure control system. The method is based on the construction of a two-sided decision mechanism, the boundary of which is described by a function called switching function. Particularly for continuous time systems, the design procedure is well established and many aspects of the control technique have

This work is supported by TOBB ETÜ BAP Program (Contract No: ETÜ BAP 2006/04)

${ }^{1}$ Corresponding Author, TOBB Economics and Technology University, Department of Electrical and Electronics Engineering, Sögütözü, Ankara, Turkey, E-mail: onderefe@ieee.org, Phone: +90-312-292-4064, Fax: +90-3122924091 already been analyzed in detail in [1], [2], [3], [4]. Variable structure control, also known as the sliding mode control, has many successful applications in chemical process engineering. See for example [5], where the process is modeled by a partial differential equation, [6], where the design is based on a first order model including dead time, and [7], where a second order sliding mode control is performed after feedback linearization, and [8], where the system is driven toward the sliding regime by an appropriate tuning of controller parameters.

One fact in all these studies needs emphasis: The sliding mode controller drives the system toward the sliding manifold and maintains the evolution on that loci, which is stable by the design and the error converges the origin of the phase space. Once the trajectories are confined to the sliding manifold, the control system displays some degrees of robustness against disturbances and parameter variations in system dynamics. This response is called invariance property of sliding mode control, [1], [9].

The motivation for utilizing sliding mode control technique in this study is the presence of noise, disturbances and uncertainties together with demanding performance expectations such as precise tracking and good management of the coolant temperature input. Despite these conditions, the available entity is a nominal dynamical model for the process under investigation.

Overall, the contributions of this paper are to define an intermediate variable that makes it easy to design a sliding mode controller and to postulate a fairly simple robust control algorithm for chemical process engineering. This paper is organized as follows: The second section introduces the nominal model of the plant, the third section presents the design of the control law. After that the simulations are presented on the true system and concluding remarks are given at the end of the paper.

\section{Nominal Model of the Process}

The process considered in this study has two state variables, namely, the dimensionless concentration denoted by $c_{1}$ and the dimensionless temperature denoted by $c_{2}$. The dynamics of the nominal process are characterized by two differential equations as given below.

$$
\begin{gathered}
\dot{c}_{1}=-c_{1}+\alpha_{n} f \\
\dot{c}_{2}=-c_{2}+\phi_{n} \alpha_{n} f-\gamma_{n}\left(c_{2}-w\right)
\end{gathered}
$$


where

$$
f\left(c_{1}, c_{2}\right):=\left(1-c_{1}\right) \exp \left(\frac{c_{2}}{1+\frac{c_{2}}{\beta_{n}}}\right)
$$

and $\alpha_{n}=0.036$ is the Damkohler number, $\gamma_{n}=25.2$ is the dimensionless cooling rate, $\phi_{n}=21.5$ is the dimensionless heat of reaction and $\beta_{n}=28.5$ is the dimensionless activation energy. The control problem is to enforce the dimensionless concentration to follow a desired trajectory by altering the dimensionless coolant temperature $(w)$. The sole constraint on the state carrying the temperature information is bounded evolution during the course of operation.

In Fig. 1, the equilibrium states are shown. According to the numerical computations based on the nominal model, the equilibrium points turn out to be stable for all equilibrium points shown in the figure. In [10], the nominal operating point of the system is described as $c_{1}=0.4126, c_{2}=3.28$ and $w=3.04$.

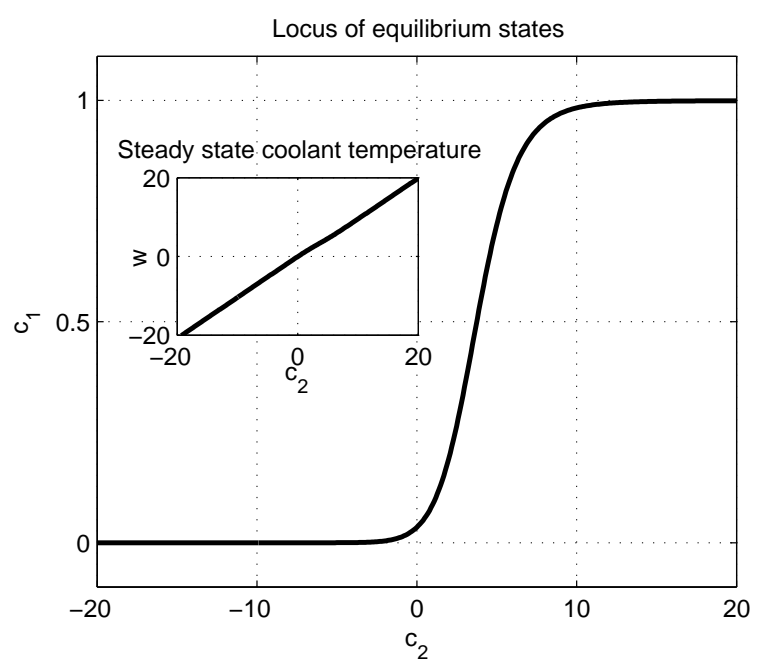

Fig. 1. Location of the equilibrium states. The window plot shows that the equilibrium coolant temperature is very close to the line $w=c_{2}$.

\section{Sliding Mode Controller Design}

The main idea in the design is to manipulate $w$ to force the value of $f(\cdot, \cdot)$ to a desired value that forces the concentration variable towards a desired value. For this purpose define this quantity as the fictitious output of the system as given below

$$
y=f\left(c_{1}, c_{2}\right)
$$

The desired value of the output is given as

$$
y_{d}=\frac{1}{\alpha_{n}} r
$$

where $r$ is the desired trajectory to be followed by $c_{1}$. Clearly if $y=y_{d}$ is maintained by some sequence of coolant temperatures, one obtains $\dot{c}_{1}=-c_{1}+r$, i.e. $c_{1}$ is forced to track $r$. Define the switching function as $s:=y-y_{d}$, and adopt the reaching law approach in [1]. Choose the coolant temperature such that the reaching dynamics in (5) is forced.

$$
\dot{s}=-\zeta \operatorname{sgn}(s)-\eta s
$$

Note that $\dot{s} s=-\zeta|s|-\eta s^{2}$, which is negative if $s \neq 0$, i.e. all trajectories in the defined phase space are attracted by the subspace described by $s=0$. Now compute $\dot{s}$ as follows:

$$
\begin{aligned}
\dot{s} & =\dot{y}-\dot{y}_{d} \\
& =\frac{\partial f}{\partial c_{1}} \dot{c}_{1}+\frac{\partial f}{\partial c_{2}} \dot{c}_{2}-\dot{y}_{d}
\end{aligned}
$$

Utilizing (1a-b) and (7a-b) in (6) and solving for $w$ yields the control law in (8).

$$
\begin{gathered}
\frac{\partial f}{\partial c_{1}}=-\exp \left(\frac{c_{2}}{1+\frac{c_{2}}{\beta_{n}}}\right) \\
\frac{\partial f}{\partial c_{2}}=\frac{1}{\left(1+\frac{c_{2}}{\beta_{n}}\right)^{2}} \\
w=\frac{-\zeta \operatorname{sgn}(s)-\eta s+\frac{\dot{r}}{\alpha_{n}}-P\left(c_{1}, c_{2}\right)}{\gamma_{n} Q\left(c_{1}, c_{2}\right)}+ \\
\frac{\left(1+\gamma_{n}\right) c_{2}-\phi_{n} \alpha_{n} f}{\gamma_{n}}
\end{gathered}
$$

where

$$
\begin{gathered}
P\left(c_{1}, c_{2}\right)=-\exp \left(\frac{c_{2}}{1+\frac{c_{2}}{\beta_{n}}}\right)\left(-c_{1}+\alpha_{n} f\right) \\
Q\left(c_{1}, c_{2}\right)=\frac{f}{\left(1+\frac{c_{2}}{\beta_{n}}\right)^{2}}
\end{gathered}
$$

With the control law in (8), the dynamics in (5) is forced. Any nonzero initial value of $s(0)$ hits the sliding subspace characterized by $s=0$ in finite time. The time of hitting is denoted by $t_{h}$ and an upper bound to which can be obtained after straightforward manipulations. More explicitly, at the time of hitting $s\left(t_{h}\right)=0$ and $\dot{s}\left(t_{h}\right)=0$ and we have

$$
s\left(t_{h}\right)=s(0)-\int_{0}^{t_{h}} \zeta \operatorname{sgn}(s(\tau))+\eta s(\tau) \mathrm{d} \tau
$$

The quantity $\operatorname{sgn}(s(t))$ is constant and does not change sign until the time of hitting. Therefore we have

$$
s\left(t_{h}\right)=s(0)-\operatorname{sgn}(s(0)) \int_{0}^{t_{h}} \zeta+\eta|s(\tau)| \mathrm{d} \tau=0
$$

Or alternatively

$$
\operatorname{sgn}(s(0))\left(|s(0)|-\int_{0}^{t_{h}} \zeta+\eta|s(\tau)| \mathrm{d} \tau\right)=0
$$

Paraphrasing and rearranging (10) yields

$$
|s(0)|=\int_{0}^{t_{h}} \zeta+\eta|s(\tau)| \mathrm{d} \tau \geq \int_{0}^{t_{h}} \zeta \mathrm{d} \tau=\zeta t_{h}
$$

which indicates $t_{h} \leq \frac{|s(0)|}{\zeta}$ sec. 


\section{TRUe System, Operating CONDitions AND SIMULATIONS}

In the validation phase, we consider the dynamical system given in (14a-b), where the state variables $x_{1}$ and $x_{2}$ correspond to the concentration and temperature, respectively. The measurements are noisy and the relevant expressions are given in $(14 c-d)$.

$$
\begin{gathered}
\dot{x}_{1}(t)=-x_{1}(t)+\alpha(t) f\left(x_{1}(t), x_{2}(t), \beta(t)\right)+d_{1}(t) \\
\dot{x}_{2}(t)=-x_{2}(t)+\phi(t) \alpha(t) f\left(x_{1}(t), x_{2}(t), \beta(t)\right) \\
-\gamma(t)\left(x_{2}(t)-w(t)\right)+d_{2}(t) \\
c_{1}(t)=x_{1}(t)+\varepsilon_{1}(t) \\
c_{2}(t)=x_{2}(t)+\varepsilon_{2}(t)
\end{gathered}
$$

where

$$
\begin{aligned}
\alpha(t) & =\alpha_{n}+\varepsilon_{\alpha}(t) \\
\beta(t) & =\beta_{n}+\varepsilon_{\beta}(t) \\
\gamma(t) & =\gamma_{n}+\varepsilon_{\gamma}(t) \\
\phi(t) & =\phi_{n}+\varepsilon_{\phi}(t)
\end{aligned}
$$

Aside from the noisy nature of the measured quantities, the process parameters are changing. As shown in Figure 2, Damkohler number, the dimensionless activation energy, dimensionless cooling rate and the dimensionless heat of reaction are time varying.
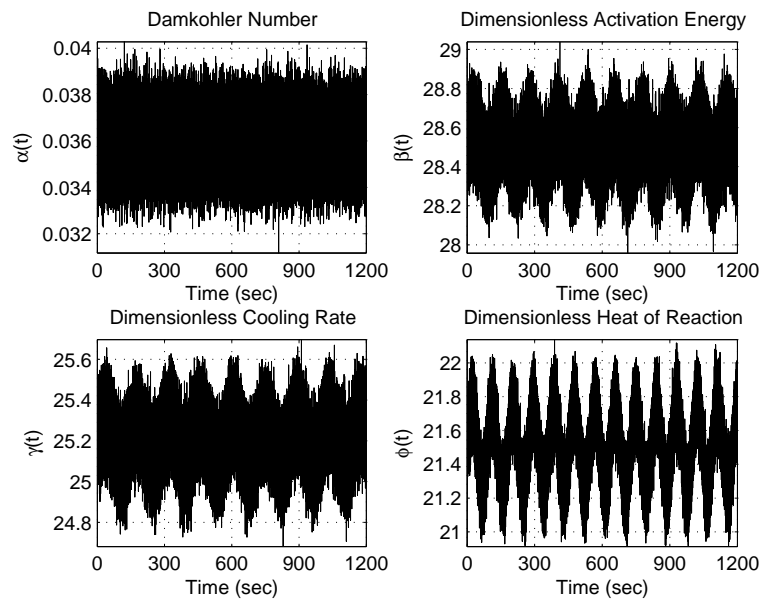

Fig. 2. Time variation in the process parameters

Although the considered bioreactor is not a completely realistic model of any biochemical process, the conditions described above make it necessary to design a robust control scheme that alleviates the difficulties stemming from the aforementioned uncertainties. Lastly, one should notice that the disturbance terms denoted by $d_{1}$ and $d_{2}$ influence the process dynamics directly (See (14a-b)). The statistical properties of the disturbance terms are summarized in Table I, where we see that the deviation from the nominal process model given in $(1 \mathrm{a}-\mathrm{b})$ is significant.
TABLE I

STATISTICAL PROPERTIES OF THE DISTURBANCES

\begin{tabular}{|c|c|}
\hline Variable & Variance \\
\hline \hline$\varepsilon_{\alpha}(t)$ & $1 e-6$ \\
\hline$\varepsilon_{\beta}(t)$ & 0.015 \\
\hline$\varepsilon_{\gamma}(t)$ & 0.015 \\
\hline$\varepsilon_{\phi}(t)$ & 0.041 \\
\hline$d_{1}(t)$ & $1 e-4$ \\
\hline$d_{2}(t)$ & $1 e-4$ \\
\hline$\varepsilon_{1}(t)$ & $1 e-5$ \\
\hline$\varepsilon_{2}(t)$ & $1 e-5$ \\
\hline
\end{tabular}

The settings of the simulation are given in Table II and the results are illustrated in Figure 3. According to the top subplot of this figure, the reference profile claims the management of the control input for three different types of desired concentration profiles, namely, a trapezoidal profile followed by a sinusoidal profile and finally a profile having step changes. This choice of the reference signal is deliberate to unfold the performance of the closed loop control system under different types of command signals that are likely to occur in practice.

TABLE II

SIMULATION SETTINGS

\begin{tabular}{|c|c|}
\hline$\zeta$ & 10 \\
\hline$\eta$ & 10 \\
\hline$\delta$ & 0.05 \\
\hline$\Delta t$ & $10 \mathrm{msec}$. \\
\hline$T$ & $1200 \mathrm{sec}$. \\
\hline
\end{tabular}

The concentration $\left(c_{1}\right)$ response of the system is plotted with the reference signal $r$ and it is seen that the two curves are almost indistinguishable. The middle subplot of Figure 3 shows the difference $r(t)-c_{1}(t)$, which is the tracking error in the concentration. The results are quite promising as the error is maintained around zero. The instant spikes due to the step change instants in the reference signal are stable.

The coolant temperature (control signal) produced by the designed controller is depicted in Figure 4, where the smoothness of the signal is one prominent feature that needs emphasis. Aside from the smoothness, one sees that the quantity $Q$ defined in (9b) must be nonzero for obtaining a physically meaningful control signal. This requires $f(\cdot, \cdot) \neq 0$, i.e. $c_{1} \neq 1$. Consequently, the desired profile $r$ should be below unity.

The behavior of the control signal during the reaching phase is illustrated in the top subplot of Figure 4, where a very quick convergence is visible. The middle subplot of the figure shows the time evolution of the fictitious output upon which the design is based. The results obtained stipulate that the fictitious process output tracks the prescribed command profile $y_{d}(t)$ very closely and as a result of this, the dynamics in $c_{1}$ resembles to $\dot{c}_{1}=-c_{1}+r$, which was the goal in this study.

In the simulations, $c_{1}(0)=0.98, c_{2}(0)=3.28$, which implies $y(0)=0.3789$. The desired value of the fictitious output is $y_{d}(0)=\frac{r(0)}{\alpha_{n}}=\frac{0.5}{0.036}=13.8889$. According to these values, we compute $s(0)=13.51$ and $t_{h} \leq 1.351$ sec. Checking this result with the observed quantities, we see $t_{h} \approx 0.1 \mathrm{sec}$, which satisfies the inequality for the upper 

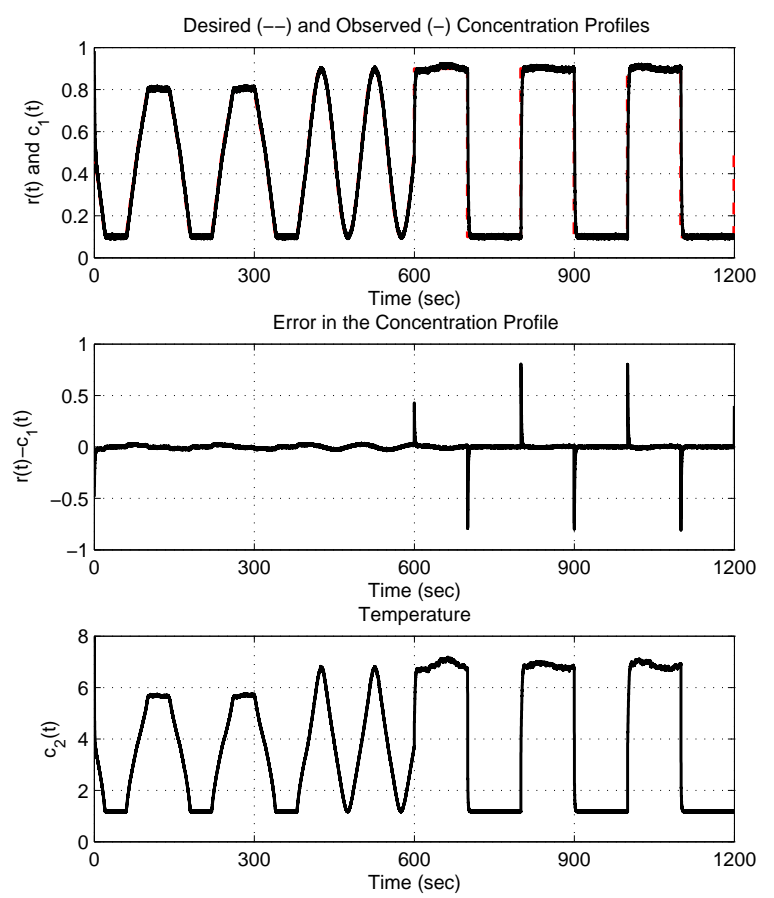

Fig. 3. The behavior of the concentration (top), the behavior of the tracking error (middle), and the behavior of temperature (bottom)

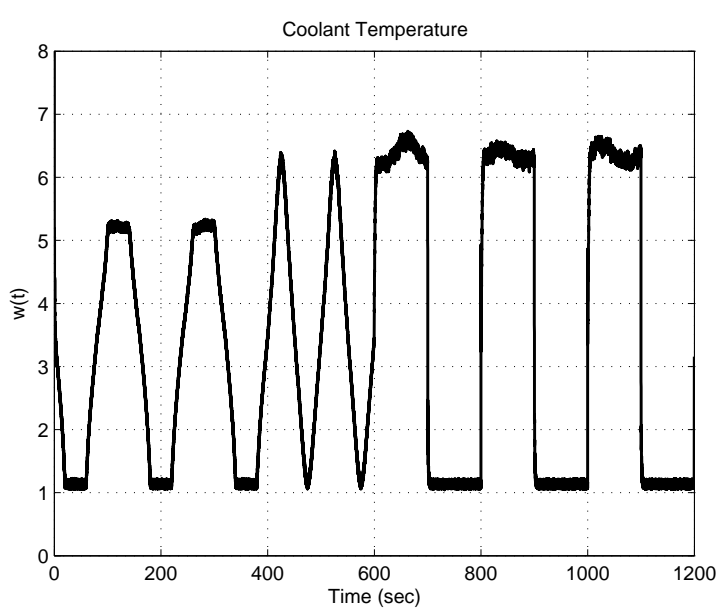

Fig. 4. The coolant rate (control signal) produced by the controller

bound on the time of hitting.

A last issue here is to figure out the extent to which the system responds robustly against the disturbances and uncertainties. For this reason, rewrite (5) with the dynamics in (14) as follows:

$$
\dot{s}=-\zeta \operatorname{sgn}(s)-\eta s+\Sigma
$$

where $\Sigma$ is a function of the state variables and disturbance terms and it it represents the deviation from the nominal dynamics collectively. It is very well known that the sliding subspace defined by $s=0$ is an attractor, i.e. $s \dot{s}<0$ is satisfied if $|\Sigma|<\zeta$ holds true. This point out to an inherent
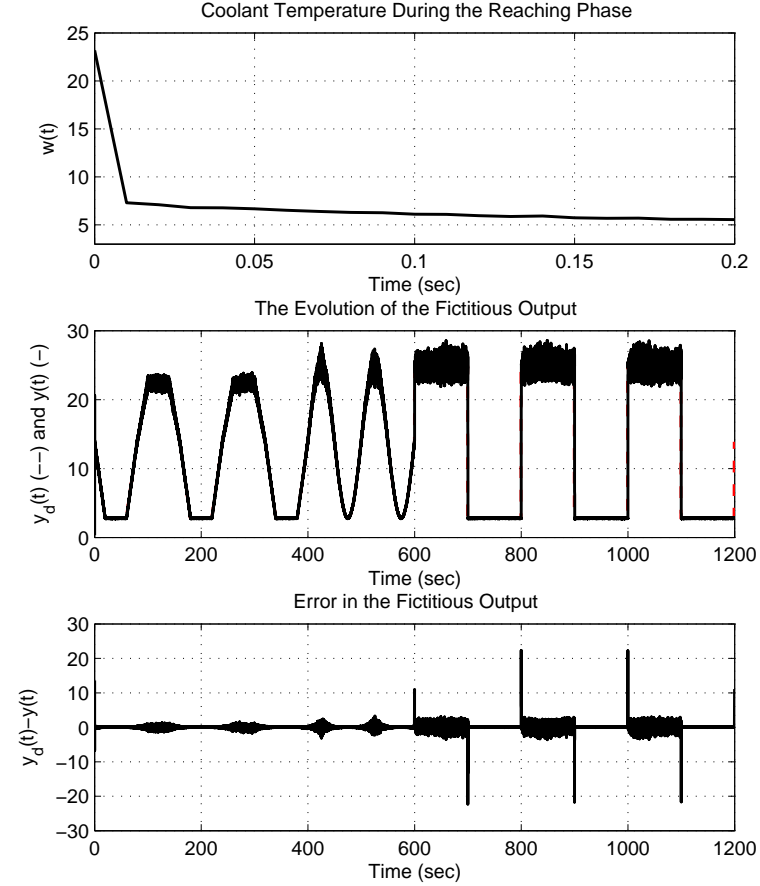

Fig. 5. Time evolution of the coolant temperature during the initial phase of the simulation (top), tracking of the intermediate variable $y$ (middle), tracking error in the intermediate variable (bottom)

tradeoff such that choosing large $\zeta$ widens the robustness margin, on the other hand, it multiplies the switching element and large values of this parameter causes undesired fluctuations that can drive the system toward instability. In our case, the chosen values of $\zeta$ assure this condition without provoking any undesired oscillation in the state variables.

The tests carried out have demonstrated that the performance is tightly dependent upon the true knowledge of Damkohler number. More explicitly, the uncertainty on $\alpha$ deteriorates the closed loop performance significantly.

\section{CONCLusions}

This paper considers the sliding mode control of biochemical process. The sliding mode control technique is very well known for its robustness against disturbances and insensitivity to parameter variations. The problem setting in this study entails such a robust controller ensuring the performance and stability. The nominal representation of the plant dynamics is assumed to be available and the design is based on this model. An intermediate variable is defined as the fictitious plant output and it is shown that if this variable tracks a suitably scaled value of the reference signal than the concentration follows the desired trajectory while assuring bounded evolution in the temperature state. During the validation phase, significant changes are considered for unfolding the robustness property of the proposed controller. Variations in the system parameters are considered together with disturbances. The results have shown that the controller performs very well under the chosen operating conditions. Smoothness of the control signal and 
the simplicity of the design are found to be the desirable characteristics of the controller.

\section{REFERENCES}

[1] Hung JY, Gao W and Hung JC. Variable structure control: a survey. IEEE Trans. on Industrial Electronics 1993; 40 (1); 2-22.

[2] Young KD, Utkin VI, and Ozguner U. A control engineer's guide to sliding mode control. IEEE Trans. on Control Systems Technology 1999; 7 (3); 328-342.

[3] Utkin VI. Sliding Modes in Control Optimization, Springer Verlag: New York, 1992.

[4] Slotine JJE and Li W. Applied Nonlinear Control, Prentice-Hall: New Jersey, 1991; 276-310.

[5] Hanczyc, E.M. and Palazoglu, A. (1995), "Sliding Mode Control of Nonlinear Distributed Parameter Chemical Processes," Ind. Eng. Chem. Res., v.34, pp.557-566.

[6] Camacho, O. and Rojas, R. (2000), "A General Sliding Mode Controller for Nonlinear Chemical Processes," Trans. of the ASME: Journal of Dynamic Systems, Measurement and Control, v.122, pp.650-655.

[7] Colantonio MC, Desages AC, Romagnoli JA and Palazoglu A. Nonlinear control of a CSTR: disturbance rejection using sliding mode control. Ind. \& Eng. Chemistry Res. 1995; 34; 2383-2392.

[8] Dagci OH, Efe MÖ, Kaynak O and Yu X, "Variable Structure Systems in Control of Biochemical Processes," Proc. of the 2001 IEEE Int. Symposium on Industrial Electronics (ISIE 2001), June 12 - 16, Pusan, Korea, v.3, pp.1690-1695, 2001.

[9] O. Kaynak, Harashima, F. and Hashimoto, H. (1984), "Variable Structure Systems Theory, as Applied to Sub-time Optimal Position Control With an Invariant Trajectory," Trans. Inst. Elect. Eng. Jpn. E, 104, pp.4752.

[10] Knapp TD, Budman HM and Broderick G. Adaptive control of a CSTR with neural network model. Journal of Process Control 2001; 11; 53-68.

[11] Khalil HK (2002). Nonlinear systems, Prentice-Hall, 3rd Ed. 